A. Ramachandran $\cdot$ C. Snehalatha $\cdot$ S. Mary $\cdot$

B. Mukesh · A. D. Bhaskar • V. Vijay •

Indian Diabetes Prevention Programme (IDPP)

\title{
The Indian Diabetes Prevention Programme shows that lifestyle modification and metformin prevent type 2 diabetes in Asian Indian subjects with impaired glucose tolerance (IDPP-1)
}

Received: 20 August 2005 / Accepted: 18 October 2005 / Published online: 4 January 2006

C) Springer-Verlag 2006

\begin{abstract}
Aims/hypothesis: Lifestyle modification helps in the primary prevention of diabetes in multiethnic American, Finnish and Chinese populations. In a prospective community-based study, we tested whether the progression to diabetes could be influenced by interventions in native Asian Indians with IGT who were younger, leaner and more insulin resistant than the above populations. Methods: We randomised 531 (421 men 110 women) subjects with IGT (mean age $45.9 \pm 5.7$ years, BMI $25.8 \pm 3.5 \mathrm{~kg} / \mathrm{m}^{2}$ ) into four groups. Group 1 was the control, Group 2 was given advice on lifestyle modification (LSM), Group 3 was treated with metformin (MET) and Group 4 was given LSM plus MET. The primary outcome measure was type 2 diabetes as diagnosed using World Health Organization criteria. Results: The median follow-up period was 30 months, and the 3-year cumulative incidences of diabetes were $55.0 \%, 39.3 \%, 40.5 \%$ and $39.5 \%$ in Groups $1-4$, respectively. The relative risk reduction was $28.5 \%$ with LSM (95\% CI 20.5-37.3, $p=0.018$ ), $26.4 \%$ with MET (95\% CI 19.1-35.1, $p=0.029$ ) and $28.2 \%$ with LSM + MET (95\% CI 20.3-37.0, $p=0.022$ ), as compared with the control group. The number needed to treat to prevent one incident case of diabetes was 6.4 for LSM, 6.9 for MET and 6.5 for LSM + MET. Conclusions/interpretation: Progression of IGT to diabetes is high in native Asian Indians. Both LSM and MET significantly reduced the incidence of diabetes in
\end{abstract}

Electronic Supplementary Material Supplementary material is available for this article at http://dx.doi.org/10.1007/s00125-0050097-z

\footnotetext{
A. Ramachandran $(\bowtie) \cdot$ C. Snehalatha $\cdot$ S. Mary

B. Mukesh · A. D. Bhaskar · V. Vijay

Diabetes Research Centre,

M.V. Hospital for Diabetes,

WHO Collaborating Centre for Research,

Education and Training in Diabetes,

Royapuram, Chennai, India

e-mail: ramachandran@vsnl.com

Tel.: +91-44-25954913

Fax: +91-44-25964938
}

Asian Indians with IGT; there was no added benefit from combining them.

Keywords Asian Indians · Diabetes prevention · Impaired glucose tolerance $\cdot$ Lifestyle modification $\cdot$ Metformin

Abbreviations DPP: Diabetes Prevention Program . DPS: Finnish Diabetes Prevention Study .

HOMA-IR: Homeostasis model assessment for insulin resistance - IDPP: Indian Diabetes Prevention Programme . LSM: lifestyle modification - MET: metformin

\section{Introduction}

Prospective randomised controlled studies such as the Diabetes Prevention Program (DPP) in the USA [1], the Finnish Diabetes Prevention Study [2] (DPS), the Da Qing IGT and Diabetes Study in China [3] and the Malmo study in Sweden [4] have shown that lifestyle modification (LSM) involving diet and enhanced physical activity helps to delay or prevent the progression of IGT to diabetes. Pharmacological agents such as metformin [1], troglitazone $[5,6]$ and acarbose [7] have also been found to be effective. Developing nations, especially India, face an enormous burden from a high prevalence of diabetes $[8,9]$; therefore, primary prevention is desirable in these countries.

The prevalence of IGT is high in native Asian Indians [8, 10] but the rate of progression to diabetes is not known. The Indian population has several unique features, including a young age of onset of diabetes, a relatively lower BMI, with high rates of insulin resistance and lower thresholds for the risk factors for diabetes [11]. The benefits of lifestyle modification in subjects with IGT in such a scenario are unknown. The usefulness of an insulin sensitiser, e.g. metformin, as a single preventive agent or as an adjunct to lifestyle modification also needs to be studied.

To date, the efficacy of interventions for the prevention of diabetes has not been investigated in native Asian Indian subjects. In the DPP study [1], 4.4\% of the study subjects 
were Asians and lifestyle modification was as effective in preventing diabetes in them as in other ethnic groups; however, these findings need to be tested in native Asian Indians. The results of the Chinese study may not be applicable to Asian Indians because Chinese individuals have a lower prevalence of diabetes and are less insulin resistant than Indians [3].

We therefore conducted this prospective randomised controlled trial in urban Asian Indian subjects with persistent IGT (IGT on two OGTT) with the aim of determining whether the incidence of type 2 diabetes could be modified by interventions.

\section{Subjects and methods}

\section{Study design}

A randomised, controlled clinical trial was performed in subjects who were diagnosed as having IGT according to the criteria of the World Health Organization [12] on two occasions (defined as persistent IGT). This was a community-based prospective study, carried out over 3 years. The study groups were as follows: Group 1 subjects were given standard health care advice (control), Group 2 subjects followed lifestyle modification (LSM), Group 3 subjects were treated with metformin (MET), and Group 4 subjects were given LSM plus MET. It was assumed that the cumulative incidence of diabetes in 3 years would be approximately $30 \%$ in the control group and that there would be a $50 \%$ reduction with the intervention methods. The sample size required in each of the four subgroups was 134 with a type 1 error of $5 \%, 80 \%$ power, and allowing for a dropout rate of $10 \%$.

\section{Primary outcome}

The primary outcome was defined as development of diabetes, indicated by either a fasting plasma glucose of $\geq 7.0 \mathrm{mmol} / 1$ $(\geq 126 \mathrm{mg} / \mathrm{dl})$ and/or a 2-h plasma glucose concentration of $\geq 11.1 \mathrm{mmol} / 1(\geq 200 \mathrm{mg} / \mathrm{dl})$ during either a 6-monthly or an annual follow-up which was confirmed by an OGTT [12]. Subjects who tested positive for diabetes were advised medical treatment and were referred to their physicians for further follow-up.

\section{Population and recruitment}

The study subjects were recruited from the middle-class population working in service organisations and also from their families. They were identified by work-place announcements and circulars. The study protocol was approved by the ethics committee of our institution. Written informed consent was obtained from the employers and from each participant in the study.

The protocol for enrolment and the randomisation of study subjects are shown in the flow chart (Fig. 1). Non- diabetic subjects with no major illness aged 35-55 years and of both sexes, were screened from March 2001 to July 2002. At the time of recruitment and during the interim 6monthly follow-up, screening was carried out using a 2-h post-glucose capillary glucose measurement and confirmatory diagnosis was based on a standard OGTT (75 g glucose load) using plasma glucose values. A total of 10,839 subjects $(8,667$ men, 2,172 women) underwent the initial screening using a glucometer (Accu-chek Sensor, Roche Diagnostics, Mannheim, Germany).

The correlation between the glucometer and venous plasma glucose values were tested in 100 blood samples and it was found to be $r=0.91, p<0.0001$ with coefficient of variation $4.9 \%$ between the readings.

IGT was detected in 1,332 subjects $(12.3 \%)$. Among these, $1,025(77 \%)$ responded when called for a confirmatory OGTT within a week. Fasting, 30-minute and 2-h blood samples were collected in the screening area at the work place. Plasma glucose was estimated in blood samples preserved with sodium fluoride/potassium oxalate in the laboratory. Venous plasma/serum samples were stored for all other biochemical tests.

World Health Organization criteria for IGT were used (fasting glucose $<7.0 \mathrm{mmol} / 1[<126 \mathrm{mg} / \mathrm{dl}] ; 2$ - $\mathrm{h}$ glucose $7.8-11.0 \mathrm{mmol} / 1$ [140-199 mg/dl]) [12].

During the second test, details of demography, age, sex, medical history, family history of diabetes, anthropometric measurements (including height, weight, waist circumference and percentage body fat using the impedance meter (Tanita TBF-611 Body Fat Monitor; Syscon Instruments, Bangalore, India), occupation and family income were recorded by standard procedures described in an earlier publication [10]. BMI was calculated. Details of blood pressure and presence of hypertension (systolic and diastolic blood pressure $\geq 140 / \geq 90 \mathrm{mmHg}$ or receiving treatment) were recorded. Annually, OGTTs, and anthropometric and blood pressure measurements were repeated. All subjects underwent a 12-lead electrocardiogram at baseline assessment and at annual follow-up to assess the presence or development of cardiac abnormalities. The team members included a physician, three laboratory technicians, a dietician, a social worker and a helper. They were trained to conduct all the test procedures. The measurements were repeated by the same members throughout the study, to eliminate interobserver variations.

An internal safety committee consisting of an administrator, a legal advisor and a consultant physician monitored the safe conduct of the study at 2-month intervals. Any serious adverse reactions were reviewed and appropriate remedial measures were taken.

A total of 531 subjects (421 men, 110 women) were diagnosed with IGT on both tests and were consecutively randomised in the four groups.

\section{Intervention}

Physical activity was assessed taking into account the occupation, mode of transport to work and leisure 
Fig. 1 Flow chart showing the procedure and results of screening, recruitment and randomisation of the study subjects

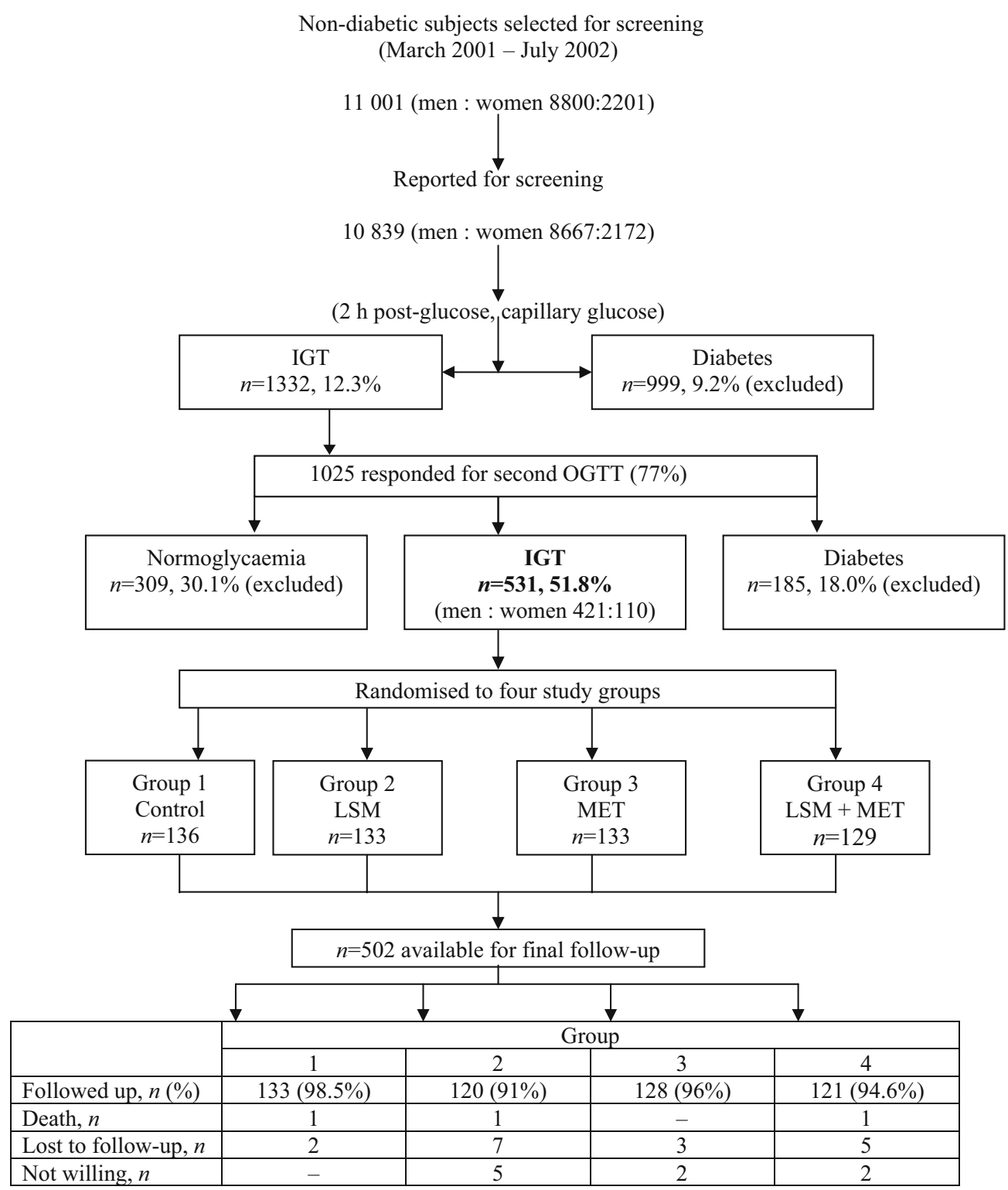

activities. The methodology has been validated in a previous epidemiological study [10].

Subjects who were involved in physical labour or who had to walk or cycle for $>30 \mathrm{~min} /$ day or were performing exercises regularly were asked to continue their routine activities. Subjects engaged in sedentary or light physical activity, as assessed in the initial interview (see Electronic supplementary material [ESM] Tables 1,2), were advised and regularly motivated to walk briskly for at least $30 \mathrm{~min}$ each day.

Subjects in the MET and LSM + MET groups received metformin tablets and were given diaries to record their daily consumption of tablets, particularly whether any doses were missed. Three months' supply was provided, and leftover tablets were counted to assess the compliance. The initial dose of $250 \mathrm{mg}$ twice daily was increased to $500 \mathrm{mg}$ twice daily in the first 50 patients after 2 weeks (26 and 24 in the MET and LSM + MET groups, respectively). Many subjects $(45 \%)$ complained of symptoms of hypo- glycaemia, such as excess hunger, giddiness and excess sweating, which were relieved by eating food. The median period of use of $500 \mathrm{mg}$ metformin twice daily was 40 days. Thereafter, all subjects in the MET and LSM + MET groups received only $250 \mathrm{mg}$ of metformin twice daily.

LSM included advice on healthy diet and regular physical activity. Scores were given based on the subjects' adherence (see ESM Tables 2, 3). Diet modification was advised for each subject, which included reduction in total calories, refined carbohydrates and fats, avoidance of sugar, and inclusion of fibre-rich foods (see ESM Text box: Dietary recommendations).

\section{Motivation and adherence}

The intervention procedure was explained individually at the time of randomisation, then again by telephone after 2 weeks or by letter. Thereafter, monthly telephonic contacts 
Table 1 Baseline demographic characteristics of the randomised groups

\begin{tabular}{|c|c|c|c|c|c|}
\hline \multirow[t]{2}{*}{ Characteristic } & \multirow[b]{2}{*}{ Total } & \multicolumn{4}{|l|}{ Group } \\
\hline & & Control & LSM & MET & $\mathrm{LSM}+\mathrm{MET}$ \\
\hline Total $(n)$ & 531 & 136 & 133 & 133 & 129 \\
\hline Men: women & 420:111 & $104: 32$ & $104: 29$ & $107: 26$ & $105: 24$ \\
\hline Age (years) & \multicolumn{5}{|c|}{$n$ (\% of subgroup) } \\
\hline $35-39$ & $82(15.4)$ & $26(19.1)$ & $18(13.5)$ & $21(15.8)$ & $17(13.2)$ \\
\hline $50-55$ & $164(30.9)$ & $34(25.0)$ & $43(32.3)$ & $43(32.3)$ & $44(34.1)$ \\
\hline Family history of diabetes & $263(49.5)$ & $80(58.8)$ & $68(51.1)$ & $55(41.4)$ & $60(46.5)^{\mathrm{a}}$ \\
\hline \multicolumn{6}{|l|}{ Occupation } \\
\hline Unskilled/skilled workers & $329(61.9)$ & $82(60.3)$ & $80(60.2)$ & $84(63.2)$ & $83(64.3)$ \\
\hline Executive/business class & $156(29.4)$ & $40(29.4)$ & $41(30.8)$ & $39(29.3)$ & $36(27.9)$ \\
\hline College & $142(26.7)$ & $33(24.3)$ & $43(32.3)$ & $36(27.1)$ & $30(23.3)$ \\
\hline Technical & $41(7.7)$ & $9(6.6)$ & $10(7.5)$ & $10(7.5)$ & $12(9.3)$ \\
\hline \multicolumn{6}{|c|}{ Monthly family income-Indian rupees } \\
\hline$\leq 5,000$ & $137(25.8)$ & $35(25.7)$ & $29(21.8)$ & $38(28.6)$ & $35(27.1)$ \\
\hline $5,000-10,000$ & $267(50.3)$ & $66(48.5)$ & $66(49.6)$ & $67(50.4)$ & $68(52.7)$ \\
\hline$>10,000$ & $127(23.9)$ & $35(25.7)$ & $38(28.6)$ & $28(21.1)$ & $26(20.2)$ \\
\hline Smoking & $115(21.7)$ & $36(26.5)$ & $29(21.8)$ & $23(17.3)$ & $27(20.9)$ \\
\hline Hypertension & $169(31.8)$ & $44(32.4)$ & $42(31.6)$ & $35(26.3)$ & $48(37.2)$ \\
\hline
\end{tabular}

${ }^{\mathrm{a}} p=0.031$ by ANOVA; all others $p>0.05$

Table 2 Baseline anthropometric and biochemical characteristics of the randomised groups

\begin{tabular}{|c|c|c|c|c|c|}
\hline \multirow[t]{2}{*}{ Characteristic } & & \multicolumn{4}{|l|}{ Group } \\
\hline & & Control & LSM & MET & LSM + MET \\
\hline \multirow{2}{*}{$n$} & Men & 104 & 104 & 107 & 105 \\
\hline & Women & 32 & 29 & 26 & 24 \\
\hline \multirow[t]{2}{*}{ Waist circumference $(\mathrm{cm})$} & Men & $90.8 \pm 7.5$ & $89.0 \pm 7.9$ & $89.7 \pm 9.5$ & $90.2 \pm 7.6$ \\
\hline & Women & $86.4 \pm 10.0$ & $88.0 \pm 9.7$ & $86.3 \pm 9.8$ & $86.5 \pm 8.8$ \\
\hline \multirow[t]{2}{*}{ Body fat (\%) } & Men & $31.6 \pm 5.4$ & $31.0 \pm 5.9$ & $29.9 \pm 5.5$ & $30.7 \pm 6.0$ \\
\hline & Women & $44.0 \pm 6.9$ & $42.8 \pm 7.3$ & $43.8 \pm 8.6$ & $43.2 \pm 7.5$ \\
\hline Blood pressure (mmHg) & Systolic & $124.1 \pm 16.0$ & $121.5 \pm 14.4$ & $120.7 \pm 15.9$ & $122.4 \pm 14.3$ \\
\hline Total cholesterol (mmol/1) & & $5.1 \pm 0.9$ & $5.2 \pm 0.9$ & $5.2 \pm 1.0$ & $5.1 \pm 0.9$ \\
\hline Triglycerides (mmol/l) & & $1.9 \pm 1.2$ & $2.0 \pm 1.4$ & $1.7 \pm 0.9$ & $1.8 \pm 0.9$ \\
\hline \multirow[t]{2}{*}{ IRI (pmol/l) } & Fasting & $130 \pm 62$ & $120.1 \pm 54.7$ & $121.6 \pm 68.5$ & $125 \pm 78.3$ \\
\hline & $2 \mathrm{~h}$ & $748.9 \pm 467$ & $704 \pm 424.3$ & $621.9 \pm 379$ & $662.8 \pm 426.4$ \\
\hline HOMA-IR & & $5.4 \pm 2.9$ & $4.8 \pm 2.5$ & $4.9 \pm 3.1$ & $5.0 \pm 3.2$ \\
\hline \multirow[t]{2}{*}{ Diet (kcal) } & Men & $2175.7 \pm 383.6$ & $2189.6 \pm 399$ & $2151 \pm 430.8$ & $2141.9 \pm 320$ \\
\hline & Women & $1841.7 \pm 237.8$ & $1974.5 \pm 353$ & $1835.6 \pm 342.9$ & $2010 \pm 373$ \\
\hline
\end{tabular}

Values are means \pm SD. Intergroup comparisons gave $p$ values $>0.05$ for all parameters by ANOVA 
Fig. 2 Cumulative incidence of diabetes, calculated using the Cox proportional hazards model. The number of subjects who underwent an annual OGTT were 484, 403 and 345 at 12,24 and 30/36 months, respectively. Rates of progression to diabetes were as follows: control $($ green line $)=55 \%$, LSM (turquoise line) $=39.3 \%$, MET $($ red line $)=40.5 \%, \mathrm{LSM}+$ MET $($ blue line $)=39.5 \%$. The $p$ values for relative risk reduction were as follows: $\mathrm{LSM}=0.018$, $\mathrm{LSM}+\mathrm{MET}=0.022$, MET $=$ 0.029. LSM and LSM + MET showed identical results, therefore, the graphs overlap

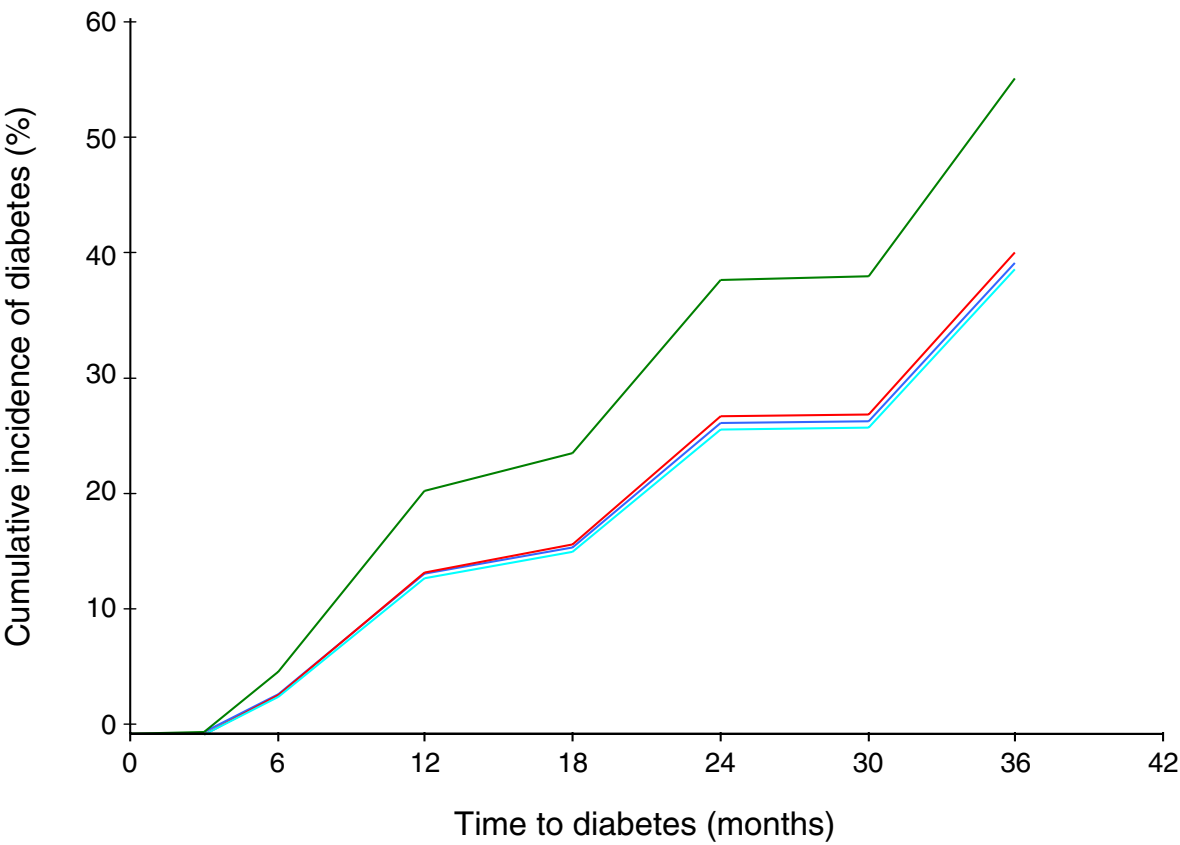

were maintained for continued motivation. Personal sessions were conducted at 6-monthly intervals.

Non-diabetic subjects in all groups underwent annual reviews for 3 years involving OGTT and measurement of anthropometric and biochemical variables. Onset of diabetes was investigated at 6-monthly intervals. At the interim 6 months, all subjects in the study were tested using a 2-h post-glucose capillary glucose test and anthropometric measurements were made. Diabetes, if detected, was confirmed by a repeat OGTT with plasma glucose estimation. Assessment of physical activity and diet adherence were made at 6-monthly reviews in both the LSM and the LSM + MET groups. Adherence to diet and physical activity was self-reported, based on weekly pattern. The average score of LSM and adherence to drug prescription were calculated at 6-monthly intervals (see ESM Tables 1,2).

Adherence to physical activity and dietary advice was computed in the LSM and LSM + MET groups (in \%). Drug adherence was calculated in the MET and LSM + MET groups. Intake of tablets was assessed by tablet count once every 3 months. If the percentage of tablets taken was
$>50 \%$ the adherence was considered to be good; others were motivated to take the tablets regularly.

Mean change in body weight $(\mathrm{kg})$ and mean change in waist circumference $(\mathrm{cm})$ at each follow-up were calculated.

\section{Biochemical assessments}

At the interim 6-month visits, glucose values were measured using glucometers, $2 \mathrm{~h}$ after a glucose load and confirmation of diabetes was made only with standard OGTT. Plasma glucose was measured using the glucose oxidase-peroxidase method. Blood samples were brought to the laboratory on ice. The fasting serum lipid profile was estimated using standard enzymatic procedures. $\mathrm{HbA}_{1} \mathrm{c}$ was measured by immunoturbidimetry (TINA-QUANT II; Roche Diagnostics Corporation, Germany); a procedure certified by the National Glycohemoglobin Standardization Program) [13]. Biochemical assays were performed on a Hitachi 912 autoanalyser using reagents from Roche Diagnostics, with appropriate quality control methods.

Table 3 Comparison of the outcome in the intervention groups at 3 years

\begin{tabular}{|c|c|c|c|c|}
\hline \multirow[t]{2}{*}{ Variable } & \multicolumn{4}{|l|}{ Group } \\
\hline & Control & LSM & MET & LSM + MET \\
\hline$n$ & 133 & 120 & 128 & 121 \\
\hline $\begin{array}{l}\text { Cumulative incidence of diabetes at year } 3 \text {, } \\
\%(95 \% \mathrm{CI})\end{array}$ & $55.0(46.0-63.5)$ & $39.3(30.4-48.5)$ & $40.5(32.0-49.7)$ & $39.5(30.9-48.9)$ \\
\hline Absolute risk reduction, $\%$ & - & 15.7 & 14.5 & 15.5 \\
\hline Relative risk reduction, \% $(95 \% \mathrm{CI})$ & - & $28.5(20.5-37.3)$ & $26.4(19.1-35.1)$ & $28.2(20.3-37.0)$ \\
\hline $\begin{array}{l}p \text { value vs control group } \\
\text { (Cox's regression equation) }\end{array}$ & - & 0.018 & 0.029 & 0.022 \\
\hline $\begin{array}{l}\text { Number needed to treat for } 3 \text { years to prevent } \\
\text { diabetes in one case }\end{array}$ & - & 6.4 & 6.9 & 6.5 \\
\hline
\end{tabular}


Table 4 Results of Cox's proportional hazard model: variables that independently influence the development of diabetes are shown

\begin{tabular}{lrlr}
\hline $\begin{array}{l}\text { Variable } \\
\text { Intervention group }\end{array}$ & $\beta$ & Hazard ratio (95\% CI) & $p$ value \\
\hline LSM & -0.474 & $0.623(0.23-1.02)$ & 0.018 \\
MET & -0.429 & $0.651(0.27-1.04)$ & 0.029 \\
LSM + MET & -0.464 & $0.629(0.23-1.03)$ & 0.022 \\
2-h plasma glucose & 0.019 & $1.019(1.003-1.035)$ & 0.015 \\
Fasting insulin & 0.021 & $1.021(1.009-1.033)$ & 0.001 \\
2-h insulin & -0.005 & $0.995(0.993-0.997)$ & $<0.001$ \\
\hline
\end{tabular}

Non-significant variables: age, sex, family history of diabetes, BMI, waist circumference, fasting plasma glucose, hypertension and smoking. No significant interactions were seen between the variables

Plasma/serum samples were stored at $-70^{\circ} \mathrm{C}$ for measurement of other biochemical parameters.

Plasma insulin was measured using the Radio-Immuno Assay Kit (Diasorin, Saluggia, Italy) which had a sensitivity of $<24 \mathrm{pmol} / 1$ and intra- and interassay CVs of $<10 \%$. Insulin resistance was calculated using the homeostasis model assessment (HOMA-IR) [14]. The normal value for insulin sensitivity by the HOMA method using the same assay method was $<4.1[15]$.

An internal safety committee monitored the adverse events and safety of study protocol. The data and final outcome measures were monitored by the international monitoring committee who had looked at the results three times, i.e. when 500 subjects had completed the follow-up assessments at 12, 24 and 30 months. The principal investigators were blinded to the interim results. After a median follow-up period of 30 months, because there were significant differences in the outcome measure between the control and intervention groups, the committee recommended the termination of the study in December 2004.

Statistical analysis

Mean and standard deviation are reported for continuous variables and inter-group comparisons were tested by twotailed ANOVA. Comparison of proportions was by $\chi^{2}$ analysis. Linear regression analysis was performed to look for a correlation between weight reduction and the change in plasma glucose at different time points during the study.

The proportion of subjects developing diabetes in each group and the factors influencing the progression were estimated using Cox's proportional hazard model. The parameters included as independent variables were: intervention groups in relation to control, sex, age, family history of diabetes, BMI, waist circumference, baseline fasting and 2-h glucose and corresponding insulin values, hypertension and smoking. Prevalence of a family history of diabetes was different between the groups and therefore it was entered as an independent variable in the equation.
Fig. 3 a Mean change in body weight during the study. A significant increase in weight $(p<0.01)$ was seen in the control group at 12,24 and $30 / 36$ months, relative to the baseline value. In the LSM group, a significant increase was seen at 24 months $(p=0.035)$. The MET and LSM + MET groups did not show significant differences as compared with baseline values. b Mean change in waist circumference $(\mathrm{cm})$ during the study. Changes were not statistically significant in any group. Diamonds, control group; rectangles, LSM group; triangles, MET group; crosses, LSM + MET group
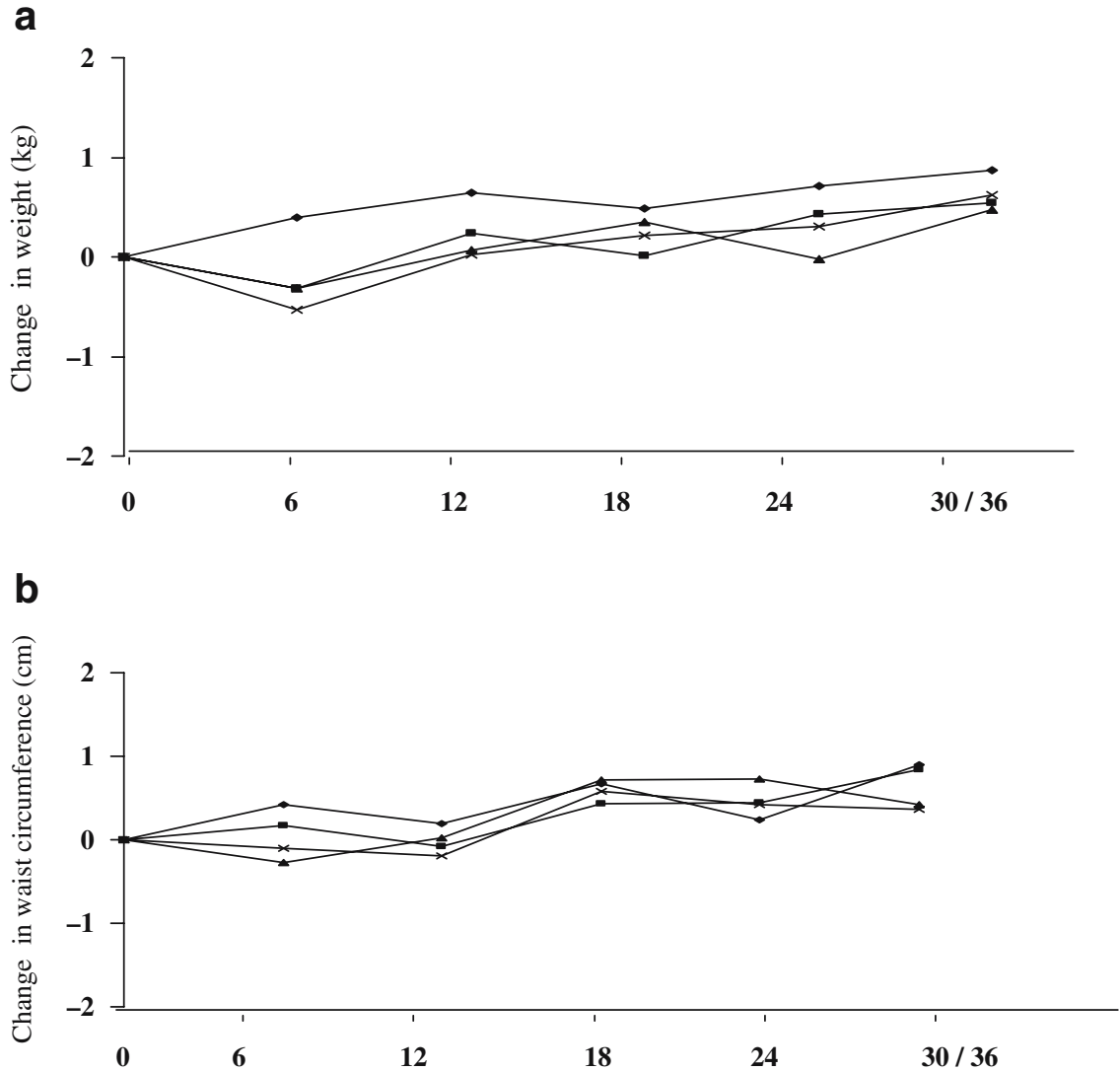

Time (months) 
Its interaction with sex, baseline values of BMI, waist circumference and 2-h plasma glucose were also tested. Interactions between age, BMI and sex were also tested.

For the intervention measures, the absolute and relative risk reductions and 95\% CIs of the estimates and the number needed to treat to prevent diabetes in one person were calculated. A $p$ value $<0.05$ was considered significant. The statistical package Stata, version 7.0 (Stata, College Station, TX, USA) was used for analyses.

\section{Results}

A total of 502 subjects underwent follow-up OGTTs. By December 2004, 213 subjects had undergone OGTTs at 36 months of follow-up. The remaining 132 subjects had completed 30 months and were recalled for a close-out OGTT in January 2005. The median follow-up at that time was 30 months.

The overall response rate at the time of the close-out OGTT was $95.1 \%$, the group-wise break up is shown in the flow chart (Fig. 1). In the subjects available for final analysis $(n=502)$ (397 men, 105 women) $44.4 \%$ had developed diabetes.

The baseline demographic characteristics (Table 1) and the anthropometric and biochemical characteristics (Table 2) were similar in the four cohorts $(n=531)$. The groups had similar educational status, occupation and prevalence of hypertension. A positive family history of diabetes, hypertension or cardiovascular disease was present in $263(49.5 \%), 177(33.3 \%)$ and $72(13.6 \%)$ subjects, respectively.

The cumulative incidence of diabetes was $55 \%$ in 3 years in control group, and it was significantly lower in all three intervention groups $(\mathrm{LSM}=39.3 \%$, MET $=40.5 \%$, LSM + MET $=39.5 \%$; Fig. 2, Table 3). The absolute and relative risk reductions with all interventions were significant (Table 3). LSM (28.5\%) and LSM + MET (28.2\%) showed similar relative risk reductions while MET showed a slightly lower risk reduction (26.4\%). The numbers to be treated to prevent one incident case of diabetes were also similar in LSM (6.4) and LSM + MET (6.5). The number was 6.9 for MET.

In the Cox's proportional hazard model, the variables that showed independent influence on the development of diabetes were baseline 2-h glucose, baseline fasting and 2-h plasma insulin and all intervention groups. The significant results are shown in Table 4. None of the interactions between the tested variables were significant.

Figure 3(a) shows the mean change in body weight (in $\mathrm{kg}$ ) at different intervals of follow-up. Significant increase from the baseline values was noted in the control group at annual follow-up. Among the intervention groups, a significant increase was only seen in LSM at 24 months $(p=0.035)$.

Mean change in waist circumference $(\mathrm{cm})$ at each interval is shown in Fig. 3b. The changes in waist circumference were not significant in any group relative to the respective baseline values.
Weight reduction and change in plasma glucose were not significantly correlated in any of the intervention groups (data not shown).

Figure 4 shows the adherence to diet, physical activity and metformin in the respective intervention groups. In LSM and LSM + MET improvement in diet adherence was seen. In LSM, diet adherence improved from $62.5 \%$ to an
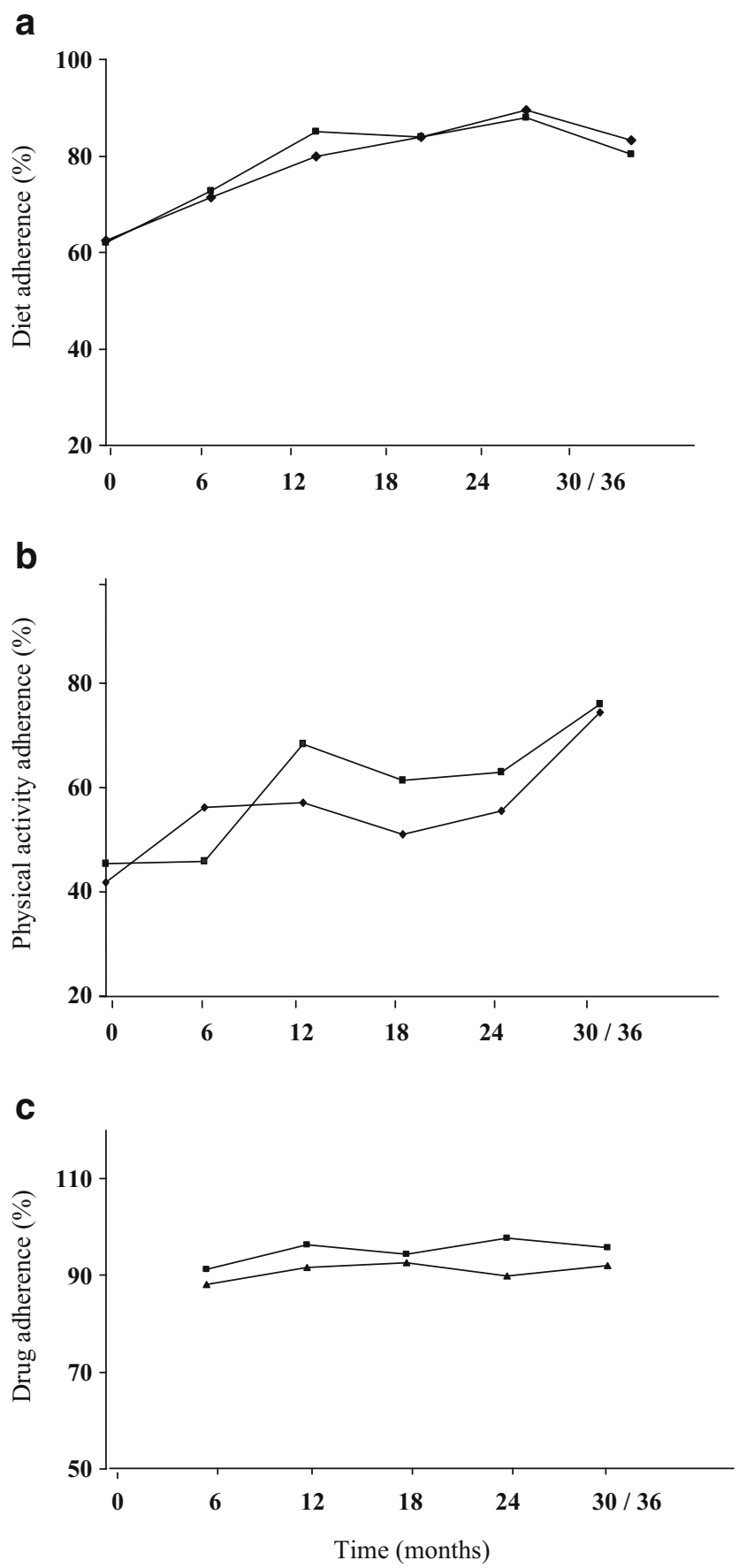

Fig. 4 Variations in adherence to diet (a), physical activity (b) and adherence to drug prescription (c). Diet and physical activity improved in the LSM (diamonds) and LSM + MET (rectangles) groups at all time points. Drug adherence was above $80 \%$ in the MET (triangles) and LSM + MET groups 
average of $81.6 \%$. In LSM + MET it improved from $62 \%$ to an average of $81.9 \%$ (Fig. 4a). Physical activity showed an improvement from $41.7 \%$ to an average of $58.8 \%$ in LSM while it increased from $45.9 \%$ to $62.9 \%$ in LSM + MET (Fig. 4b). The average percentage of drug adherence was $90.9 \%$ in MET and $95.1 \%$ in LSM + MET (Fig. 4c).

\section{Adverse events}

There were 11 cases of cardiovascular events: two in controls, four in LSM and five in LSM + MET. In the control group, one subject died following surgery for a cerebrovascular accident. In the LSM group, one subject died of hepatic encephalopathy and in the LSM + MET group one subject died during the postoperative period after thyroid surgery. There were 25 other cases of hospitalisation for various surgeries. All the subjects involved recovered uneventfully. In the MET and LSM + MET groups symptoms of hypoglycaemia were reported by 22 subjects in the MET and LSM + MET groups when they were receiving metformin $500 \mathrm{mg}$ twice a day. The symptoms were relieved after eating food. Five cases had gastrointestinal symptoms with this dose of metformin. The symptoms did not recur when the dose was reduced to $250 \mathrm{mg}$ twice a day.

\section{Discussion}

The most important observation of this intervention study was that it was possible to prevent diabetes in native Asian Indian subjects with IGT using lifestyle modification, despite their relatively low BMI and highly insulin-resistant characteristics. Metformin was also effective in a smaller dose $(500 \mathrm{mg} /$ day $)$ than used in the DPP $(1,700 \mathrm{mg} /$ day $)$ [1]. It was surprising that the effects of LSM and LSM + MET were not different. There was no additional benefit seen by combining metformin with LSM.

The progression rate of IGT to diabetes was very high in Asian Indians, as shown by a cumulative incidence of $55.0 \%$ in 3 years $(18.3 \%$ per year) in the controls. This was significantly higher than in the Finnish (6\% per year] [2], American (11 per 100 person-years) [1] and Chinese (11.3\% per year) studies [3]. The Indian, Finnish [2] and Chinese [3] cohorts had persistent IGT on recruitment. The possibility of some cases with diabetes being included was eliminated by a repeat OGTT. The high rate of progression to diabetes might be related to the low risk thresholds in Asian Indians, which were observed in several of our previous studies [11].

It is important to note that metformin in lower doses $(500 \mathrm{mg})$ was effective in reducing the progression rate of IGT to diabetes in the Asian Indian population. The absolute reduction in our study was $14.5 / 100$, which was higher than in the DPP (7.2/100) [1]. This was also seen in the lower number needed to be treated to prevent one case of diabetes (6.9) in this study compared with in the DPP
(13.9) [1]. The lower dose requirement may be related to the lower BMI of Asian Indians. The higher risk reduction in IDPP may be mostly related to the higher progression rate in the control group.

In the DPP, effectiveness of metformin and LSM were similar in Asian and other ethnic groups [1]. LSM was more effective than metformin in all races, including the ethnic minority population, and the effect of metformin was lower in the thinner individuals. The dissimilarities between the results in the native Asian Indians and those in the migrant Asian population in the DPP [1] might be related to several factors. First, the Indian study cohort consisted of a middle-class working population, many of whom were already physically active and were on a diet similar to that prescribed. Our study subjects did not lose weight. The socioeconomic factors influencing lifestyle were different in the native Asian Indian population and in the migrants in the DPP study [1]. Second, subjects in the IDPP had a high propensity to develop diabetes as they had reproducible IGT. This was apparent from a higher rate of progression seen in native Asian Indians.

No synergistic effect was noted by combining LSM and metformin. This will be explained by further analysis of the mechanisms responsible for the observed results. The relative risk reduction with LSM was less in the IDPP when compared with the DPP and the Finnish studies $(58 \%$ in both) $[1,2]$.

The Indian and Chinese cohorts [3] had similar mean age and BMI, (age Indian 45.9 \pm 5.7 years, Chinese $45 \pm 9.1$ years), BMI $\left(\mathrm{kg} / \mathrm{m}^{2}\right)$ (Indian $25.8 \pm 3.5$ and Chinese 25.8 \pm 3.8 ) but Indians had a higher progression rate compared with the Chinese. The Indians were younger and leaner compared with the Finnish [2] (age 55 \pm 7.0 years, BMI $31 \pm 4.6$ ) and the American cohorts [1] (age 50.6 \pm 10.7 years, BMI 34 \pm 6.7 ). The period of follow-up was similar in the Indian, American [1] and Finnish studies [2].

The change in body weight was not significant in our study population unlike in the DPP [1] and in the Finnish [2] studies. Mechanisms responsible for the beneficial effects of interventions, independent of weight change, need to be analysed. A recent clinic-based lifestyle intervention in Japanese men with IGT showed that the benefits of intervention could not be solely ascribed to weight reduction [16].

Our study has the limitations of not being a placebocontrolled, blinded study. However, the principal investigators were blinded to the outcome until they were asked to close the study by the international data monitoring committee. Loss to follow-up, though only a small percentage, had occurred in all subgroups.

Our study has shown that the progression of IGT to diabetes is high in the native Asian Indian population. It has also demonstrated the effectiveness that lifestyle modification involving moderate, but consistent, physical activity and diet modification help to prevent diabetes even in the Asian Indians, who have a high risk of developing diabetes. Metformin in smaller doses than those used in DPP was equally effective. Addition of metformin to LSM did not enhance the effectiveness. 
Acknowledgements We acknowledge the valuable help given by the following Indian Diabetes Prevention Programme team members in conducting the field survey: C. K. Sathish Kumar, S. Selvam, M. Muruganandham, A. Catherin Seeli, V. Lalitha Manjula and L. Subhashini. We also thank M/S US Vitamins for their financial support of the study, particularly its medical director, V. V. Joshi, for his valuable suggestions. We are grateful for the services of the members of the Institutional Safety Monitoring Committee, Steering Committee and also the International Data Monitoring Committee, who provided valuable guidance throughout the study. We thank M. Engelgau, USA; J. Tuomilehto, Finland and G. Alberti, UK, who are members of the International Data Monitoring Committee, and K. Satyavani for being the local representative for the committee. We thank B. Sitaraman, A. $\mathrm{S}$. Ranganathan and S. Abuthahir for serving as members of the Safety Monitoring Committee. The secretarial help of L. Vijaya and the technical help and laboratory estimations provided by S. Sivasankari are gratefully acknowledged. We also thank the managements of various service organisations. We are particularly indebted to all the participants of the study for their co-operation.

\section{References}

1. Knowler WC, Barrett-Connor E, Fowler SE et al (2002) Reduction in the incidence of type 2 diabetes with lifestyle intervention or metformin. Diabetes Prevention Program Research Group. N Engl J Med 346:393-403

2. Tuomilehto J, Lindstrom J, Eriksson JG et al (2001) Prevention of type 2 diabetes mellitus by changes in lifestyle among subjects with impaired glucose tolerance. N Engl J Med 344:1343-1350

3. Pan XR, Li GW, Hu YH et al (1997) Effects of diet and exercise in preventing NIDDM in people with impaired glucose tolerance: the Da Qing IGT and Diabetes Study. Diabetes Care 20:537-544

4. Eriksson KF, Lindgarde F (1997) Prevention of type 2 (noninsulin-dependent) diabetes mellitus by diet and physical exercise: the 6-year Malmo feasibility study. Diabetologia 34:891-898

5. The Diabetes Prevention Program Research Group (2005). Prevention of type 2 diabetes with troglitazone in the diabetes prevention program. Diabetes 54:1150-1156
6. Buchanan TA, Xiang AH, Peters RK et al (2002) Preservation of pancreatic beta-cell function and prevention of type 2 diabetes by pharmacological treatment of insulin resistance in high-risk Hispanic women. Diabetes 51:2796-2803

7. Chiasson JL, Josse RG, Gomis R, Hanefeld M, Karasik A, Laakso M. (2002) Acarbose for prevention of type 2 diabetes mellitus: the STOP-NIDDM randomised trial. The Lancet 359:2072-2077

8. Wild S, Roglic G, Green A, Sicree R, King H (2004) WHOGlobal prevention of diabetes: estimates for the year 2000 and projections for 2030. Diabetes Care 27:1047-1053

9. International Diabetes Federation (2003) Diabetes Atlas 2003, Brussels, International Diabetes Federation

10. Ramachandran A, Snehalatha C, Kapur A, et al (2001) For the Diabetes Epidemiology Study Group in India. High prevalence of diabetes and impaired glucose tolerance in India: National Urban Diabetes Survey. Diabetologia 44:1094-1101

11. Ramachandran A, Snehalatha C, Vijay V (2004) Low risk threshold for acquired diabetogenic factors in Asian Indians. Diab Res Clin Pract 65:189-195

12. World Health Organization (1999) Definition, Diagnosis and Classification of Diabetes Mellitus and its Complications. Report of a WHO Consultation. Part 1: Diagnosis and Classification of Diabetes Mellitus. Geneva, World Health Organization

13. National Glycohemoglobin Standardization Program. List of NGSP certified methods. Available from http://www.missouri. edu/ diabetes/ngsp/indexmbg.htm, last accessed in November 2005

14. Mathews DR, Hosker JP, Rudenski AS, Naylor BA, Treacher DF, Turner RL (1985) Homeostasis model assessment: insulin resistance and B-cell function from fasting plasma glucose and insulin concentrations in man. Diabetologia 28:412-419

15. Ramachandran A, Snehalatha C, Satyavani K, Sivasankari S, Vijay V (2003) Metabolic syndrome in urban Asian Indian adults - A population study using modified ATP III criteria. Diab Res Clin Pract 60:199-204

16. Kosaka K, Noda M, Kuzuya T (2005) Prevention of type 2 diabetes by lifestyle intervention: a Japanese trial in IGT males. Diab Res Clin Pract 67:152-162 